\title{
A Clustering Sleep Scheduling Mechanism Based on Sentinel Nodes Monitor for WSN
}

\author{
Zhan-Yang $\mathrm{Xu}^{1}$, Song-Gang Zhao ${ }^{2}$ and Zheng-Jun Jing ${ }^{3}$ \\ (Nanjing University of Information Science and Technology, college of Computer \\ and Software, Nanjing, 210044, China) \\ zsg412@126.com
}

\begin{abstract}
This paper proposes a clustering sleep scheduling mechanism based on sentinel nodes monitoring for WSN. The mechanism combines the network clustering strategy with the node dormancy strategy, and improves the method of selecting the candidate cluster heads randomly in Energy-Efficient Unequal Clustering (EEUC) algorithm. The conception of sentinel node is introduced based on EEUC, and the neighbor node set of sentinel node will be dormant when the sentinel node's data change rate is lower than the setting threshold. Simulation results show that this mechanism can effectively balance the energy consumption of the entire network, and significantly extend the network lifetime.
\end{abstract}

Keywords: WSN; Sleep; EEUC; Sentinel Nodes; Data Change Rate

\section{Introduction}

In recent years, with the further development of wireless communication technology, many researchers have done a lot of deepening research work in the field of WSN (Wireless Sensor Networks). WSN [1-4] can sense, inspect and gather the information of objects through wireless communication among the sensor nodes and finally send the information to the sink node. Sensor node is generally small, and its life cycle mainly relies on the limited energy supplied by batteries. Due to the massive spread of sensor nodes in the monitoring area, recharging the batteries frequently is not a practical option. Therefore, it has become a hot research problem to achieve high energy efficiency in wireless sensor networks.

According to the papers [5-6], the most energy consumption modules in wireless sensor networks mainly include: sensor module, processor module and data communication module. Data communication module consumes the most energy. The sensor node usually has three states: work, leisure and sleep. Energy consumption in the sleep state is far less than others. As a result, if we can design a node's working state allocation mechanism or a reliable data transport mechanism for the sensor nodes by using their local computing and storage capacity, the energy consumption of network can be reduced effectively and the life cycle of network will be prolonged obviously. In this case, under the premise of meeting the monitoring results accuracy requirement, how to make some nodes sleep temporarily has become a hot problem.

In this case, we propose a clustering sleep scheduling mechanism based on sentinel nodes monitoring for WSN. We introduce the concept of sentinel node and combine the network clustering strategy with the node sleep scheduling mechanism. The role sentinel node, which loses the election to be cluster head among the candidate cluster heads, likes sentry soldier in our protocol. The main application scenarios of our mechanism are in real-time data-aware, target tracking, and data collection, etc.

The rest of our paper is organized as follows: Section 2 introduces the related work; Section 3 defines data model related the network; Section 4 expounds the method of 
choosing candidate cluster heads, and the node sleep scheduling strategies; Section 5 presents the experimental results and analysis the performance of our mechanism; Section 6 makes a summary of our paper.

\section{Related Work}

Clustering routing in wireless sensor network has become a focus in current study of the routing technology, and compared with the traditional routing, cluster-based routing protocols are better in terms of network topology management, energy minimization as well as data aggregation. LEACH (low-energy adaptive clustering hierarchy) [7], which was put forward by Heinzelman, is the foundation in wireless sensor network routing protocol research. This protocol includes a new, distributed cluster formation technique that enables self-organization of large numbers of nodes. Then the centralized structure of cluster algorithm LEACH-C and LEACH-E [8] considering the node energy were proposed. Lindsey S proposed the PEGASIS [9], which put nodes into a chain. Sensors receive and send data by nearest neighbor nodes, finally transmit the aggregated data to the sink node by a specified one. Younis put forward the HEED [10] which uses a fixed cluster radius. They took the node residual energy and the cost of inner-cluster communications into consideration when selecting clusters heads, and spreading the network energy consumption to prolong the network life.

On this basis, Wu presented EEUC [11] algorithm. In their scheme the Sink node first broadcasts a "hello" message to all the nodes at a certain power level, by this way each node can compute the approximate distance called "d" to the Sink based on the received signal strength. The probability of each node selected as candidate cluster heads is $\mathrm{T}$, and candidate cluster heads will compute their competition radius called " $R_{c}$ " computed by equation (1):

$$
R_{c}=\left(1-c \frac{d_{\text {max }}-d\left(s_{i}, D S\right)}{d_{\max }-d_{\text {min }}}\right) R_{C}^{o}
$$

Then each candidate node checks whether there is a candidate node with more residual energy within their competition radius. Once the candidate node finds a more powerful candidate node, it will give up the competition; otherwise, it will be elected as cluster head in the end. Under these rules, there will be more clusters in the areas near the Sink node, and these clusters are smaller in size. So the cluster heads can save more energy in inner-cluster energy communication to make up the consumption generated by large amounts of data forwarding between clusters. The simulation results show the network lifetime is better than the above.

Nodes sleep scheduling [12-14] is another way to prolong the network life. RS (Randomized Scheduling) [15] is one of the Centralized sleep scheduling algorithm in which sink node informs some nodes to sleep with the probability $\mathrm{P}$ and wake up them with the probability (1-P). However, the reason why we set the sleep probability as $\mathrm{P}$ lacks the basis. LDS (Linear Distanced-based Scheduling) [16] is more fully than RS in theoretical basis. In this method, the nodes working states are controlled by two factors: one is the distance between the node and sink, the other is the maximum number of nodes allowed to be sleep. While this algorithm needs to know each node's location, it's very difficult in the practical application of wireless network. The concept of redundant nodes was proposed in DSA (Distance Superabundance Analysis) [17], in this algorithm redundant nodes will be closed on the basis of the area can be fully covered and nodes is able to communicate freely everywhere in the area. However, this algorithm is still too complicated and difficult to apply in reality.

Therefore, we put forward a sleep scheduling protocol based on nodes' data change rate, and apply this protocol to clustering routing algorithm based on EEUC, called SSEEUC (Sleep Scheduling based on Energy-Efficient Unequal Clustering). The key 
points of this protocol lie in the method of choosing the candidate cluster heads, and the node sleep scheduling strategy. We hope the candidate cluster heads can relative evenly distributed in the monitoring area. In order to achieve this goal, the remaining energy and the geographical position of nodes are fully considered in the candidate cluster heads selection phase. After completing the selection of candidate cluster head, we generate clusters among the monitoring area according to EEUC agreement, and these candidate cluster heads that loses the election to be cluster head will become the sentinel node in each cluster. Sentinel node will wake up the neighbor nodes when the data change value of it is higher than the preset threshold value $\mathrm{v}$; otherwise the neighbor nodes will keep sleeping state. The routing protocol of our mechanism is consistent with EEUC: the innercluster communication is based on single hop way, while multi-hops are applied in the data transmission from cluster heads to sink node. Simulation experiments show that the proposed algorithm can effectively balance the energy consumption of sensor nodes and prolong the lifetime of networks.

\section{Preliminaries}

In this section, we introduce the several models used for this article; in order to ensure the comparability with EEUC protocol we adopt the similar network model and energy model.

\subsection{Network Model}

We assume that the sensor network is consisted of $\mathrm{N}$ sensor nodes uniformly deployed over a vast field to continuously monitor the environment. The sensor is denoted by $s_{i}$, and the corresponding sensor node set is $S=\left\{\mathrm{s}_{1}, \mathrm{~s}_{2}, \ldots, \mathrm{s}_{n}\right\}$, where $|S|=n$. The following is about some assumptions of the model:

1. The position of each node will never change after deployed, the Sink node is out of the monitor area;

2. Every node is isomorphism and has a unique identifier (ID);

3. Node can freely adjust the transmission power to save energy according to the distance to the receiver;

4. Node can compute the approximate distance to another node based on the received signal strength, if the transmitting power is known.

\subsection{Energy Consumption Model}

Assume the distance between the transmitter and receiver is $d$, the energy spent for transmission of a $l-b i t$ packet over distance $d$ is:

$$
E_{T x}(l, d)= \begin{cases}l E_{\text {elec }}+l x_{f s} d^{2} & d<d_{0} \\ l E_{\text {elec }}+l x_{m p} d^{4} & d \geq d_{0}\end{cases}
$$

Both the free space ( $d^{2}$ power loss) and the multi-path fading ( $d^{4}$ power loss) channel models are considered in the model [18], $l E_{\text {elec }}$ is the energy consumption while node receives or transmits $l-b$ it packet.

\subsection{Node Sensing Data Model}

The SSEEUC is mainly suitable for these application scenarios such as real-time dataaware, target tracking, and environmental areas data collection, etc. In these scenarios, the data sensed by the nodes are related to their location and time. The sensing data is stable 
for most of the time and the induction of adjacent nodes in location or rounds are similar except the target objects suddenly appear in the area or regional climate change suddenly. Considering the appeal factor, we assume the sensing data values $Q_{(r, x, y)}$ of node $\mathrm{s}_{i}$ whose coordinate is $\left(x_{i}, y_{i}\right)$ in the $r-t h$ round as follow:

$$
Q_{(\mathrm{r}, x, y)}=A \sin \left[\left(w p+\frac{x_{i}^{2}+y_{i}^{2}}{x_{\max }^{2}+y_{\max }^{2}} \square_{r}\right]+B\right.
$$

$A, w, B$ are parameters in the formula, and $x_{\max }, y_{\max }$ are side length of the rectangular monitoring area.

\section{The Detail of SSEEUC Protocol}

The SSEEUC protocol consists of two parts: a relatively uniform candidate cluster head selection method and a node sleep scheduling algorithm based on the sentinel node data change rate. In order to receive a better detection result, the candidate cluster heads should be evenly distributed relatively so that the sentinel nodes can be evenly distributed relatively in the monitoring area. And these candidate cluster heads will become sentinel nodes that lose the election to be cluster heads.

\subsection{Uniform Candidate Cluster Head Selection}

At the network deployment stage, the sink node broadcasts a signal to every sensor, each node computes the approximate distance to sink node by the strength of received signal. Unlike EEUC randomly generated candidate cluster heads in probability $\mathrm{T}$, we set a competition rule as follows to choose them by considering the nodes' residual energy and location factors:

Step 1: Every sensor broadcasts a message which contains the node ID, residual energy $R_{E}$ and the mark $Z$ with the same broadcast radius $R$, The value of $Z$ is 0 or 1 , which means whether the node is elected as sentinel node or not, respectively. $R$ is determined by the proportion of candidate cluster heads in need.

Step 2: Each node receives the broadcast messages around them, and computes the distance $d$ between each other, then saves these information in a set $S_{n h}$ as its "adjacent" nodes.

Step 3: Node $s_{i}$ will determine whether it can be selected as candidate cluster heads after the other nodes which carry more residual energy make the decision, once $s_{i}$ finds itself carry the most residual energy, it will be elected and broadcast a victory message with the radius $R$.

Step 4: If $s_{i}$ receives the victory message from neighbor node $s_{j}$, it will give up the competition and broadcast a failure message with the radius $R$.

Step 5: If $s_{i}$ receives the failure message from neighbor node $s_{j}, s_{j}$ will be eliminate from the set $S_{n h}$ of $s_{i}$.

Step 6: After the candidate cluster heads are selected completely, clusters will be built with the EEUC protocol. 
Final Clusters routing is shown as figure1:

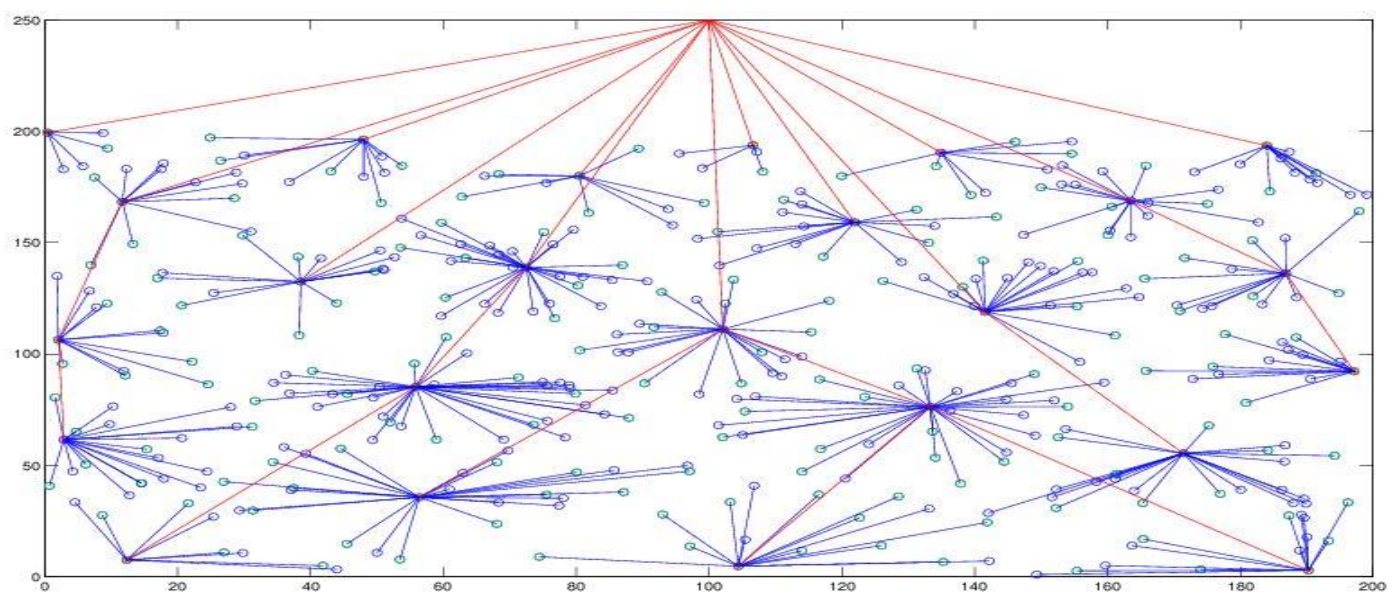

Fig. 1 The Clusters Routing Tree in SSEEUC

The following is the candidate cluster head distribution contrast figures in a random round. Due to the nodes is very density, we capture part of the images in order to get a better look:
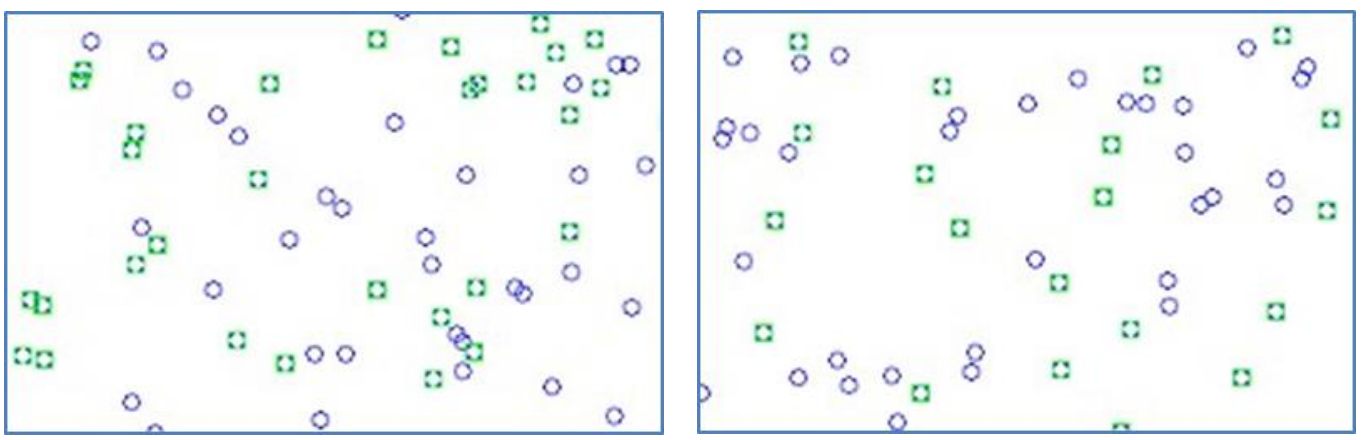

\section{Fig.2 The Node Distribution in EEUC Fig.3 The Node Distribution in SSEEUC}

In the diagram, green square nodes represent candidate cluster heads, and the purple circular nodes represent the common node. We can clearly see that the candidate cluster heads are evenly distributed in SSEEUC while some candidates always get together in EEUC.

\subsection{Sleep Scheduling Algorithm}

In wireless sensor network, different applications have different requirements of sensors' work state. Some always have new data produced while some are in the steady states for the most time. Our sleep scheduling algorithm is suitable for the latter. We should set part of the nodes in dormancy while the detection area is quiet so as to save energy. In this case, we put forward the concept of the sentinel node which is renamed by the candidate cluster head who loses the election to be cluster head. The sentinel nodes are uniform distributed in the area relatively, and have more residual energy. The function of sentinel nodes is to change the neighbor nodes' work state based on the sentinel node' data change rate between adjacent two rounds. However, each round of the clusters are refactoring, the probability of the same node successive elected candidate cluster head is not high. So we compute the data change rate by comparing the current 
data with the nearest node which was elected as sentinel last round in the set $S_{n h}$ if it exists. It will wake up the neighbor nodes in set $S_{n h}$ when the data change value is higher than the preset threshold value $\mathrm{v}$; otherwise the neighbor nodes keep a dormant state. The specific scheduling process is as follows:

Step 1: Clusters was built with the EEUC protocol, those candidate cluster heads lost the election play the sentinel node, and other nodes will sleep;

Step 2: Sentinel node $s_{i}$ get the current sensing data value as $Q$, if $s_{i}$ was sentinel node last round by checking the mark $Z$. Assume that the sensing data value is $Q_{0}$ and the predefined threshold is $v$, if $\left|Q-Q_{0}\right|>v, s_{i}$ will broadcast a wake up message to the neighbor nodes with the radius $R$ as we think there are something happening around it.

Step 3: If the mark $Z=0$, it means the node $s_{i}$ is not sentinel node last round. $s_{i}$ will look for the nearest node $s_{j}$ whose mark $Z=1$ in the set $S_{n h}$ if it exists. Assume that the sensing data value of $s_{j}$ is $Q_{1}$, if $\left|Q_{1}-Q\right|>v, s_{i}$ will broadcast a wake up message to the neighbor nodes with the radius $R$ as we think there are something happening around it.

Step 4: If $s_{j}$ of step 3 doesn't exists, $s_{i}$ will broadcast a wake up message to the neighbor nodes with the radius $R$ as we think there is something happening around it.

Step 5: The ordinary node will remain dormant if any of these above conditions are not satisfied.

The coverage of the sentinel nodes also can be adjusted by the broadcast radius $R$ in specific applications. The decrease of will lead to an increase of the sentinel nodes' number, thus the coverage will increase.

\section{Simulation Results and Analysis}

In order to make a comparison of test results, the parameters used in the SSEEUC experiment are consistent with EEUC. We find that when $R=10 \mathrm{~m}$ the candidate cluster heads number of SSEEUC and EEUC are almost same after a lot of experiments. The specific parameter values showed in Table 1:

Table 1. Simulation Parameters

\begin{tabular}{ll}
\hline Parameter & Value \\
\hline Network field & $(0,0)-(200,200) \mathrm{m}$ \\
Sink node location & $(100,250) \mathrm{m}$ \\
$\mathbf{N}$ & 400 \\
Initial energy & $0.5 \mathrm{~J}$ \\
$\boldsymbol{E}_{\text {elec }}$ & $50 \mathrm{~nJ} / \mathrm{bit}$ \\
$\xi_{\mathrm{fs}}$ & $10 \mathrm{pJ} /\left(\mathrm{bit}^{*} \mathrm{~m}^{2}\right)$ \\
$\xi_{\mathbf{m p}}$ & $0.0013 \mathrm{pJ} /\left(\mathrm{bit}^{*} \mathrm{~m}^{4}\right)$ \\
\hline
\end{tabular}




\begin{tabular}{ll}
\hline $\mathbf{d}_{\mathbf{0}}$ & $87 \mathrm{~m}$ \\
$\mathbf{E}_{\mathbf{D F}}$ & $5 \mathrm{~nJ} /(\mathrm{bit} *$ signal $)$ \\
Data packet size & $4000 \mathrm{bits}$ \\
$\mathbf{A}$ & 5 \\
$\mathbf{\omega}$ & 0.002 \\
$\mathbf{B}$ & 15 \\
$\mathbf{R}$ & $10 \mathrm{~m}$ \\
$\mathbf{v}$ & 0.5 \\
$\mathbf{c}$ & 0.5 \\
\hline
\end{tabular}

\subsection{Cluster Head Characteristics}

The stability of the cluster structure is an important measurement of the clustering protocol, and the number of cluster heads produced every rounds is a direct embodiment to the stability. Figure 4 shows the distribution of the clusters number in the one hundred rounds in LEACH, HEED, EEUC and the SSEEUC.
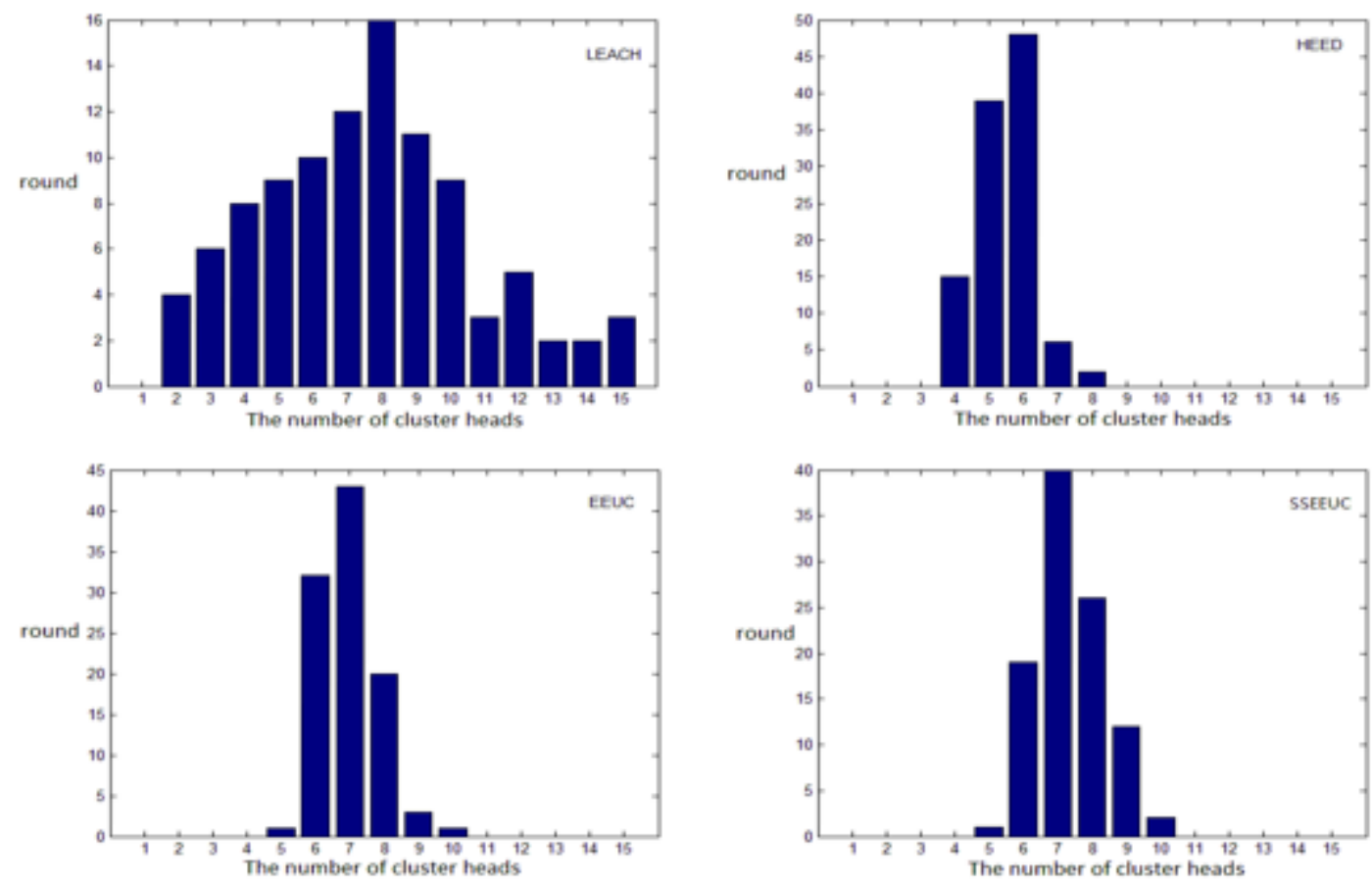

Fig.4 Distribution of the Number of Cluster Heads

We can see from the figure 4 that $\mathrm{LEACH}$ is the most unstable protocol in the distribution of the clusters number, this's because it selects the cluster heads just by the 
random value and threshold while the others considers the nodes' residual energy. The number of HEED is less than EEUC and SSEEUC because both of them take the theory of non-uniform clustering. EEUC and SSEEUC have the nearly distribution of cluster heads number as they have the similar proportion of candidate node when $R=10 \mathrm{~m}$.

\subsection{Energy Efficiency}

Due to the limited energy of senor nodes, how to use energy effectively is the core of our research. As cluster heads consume the main energy of the network in each round, we compare the energy consumption of cluster heads in a round of LEACH, HEED, EEUC and SSEEUC. Figure 5 shows the curves of the sum of the cluster heads energy consumption for the 4 protocols by choosing 20 rounds randomly.

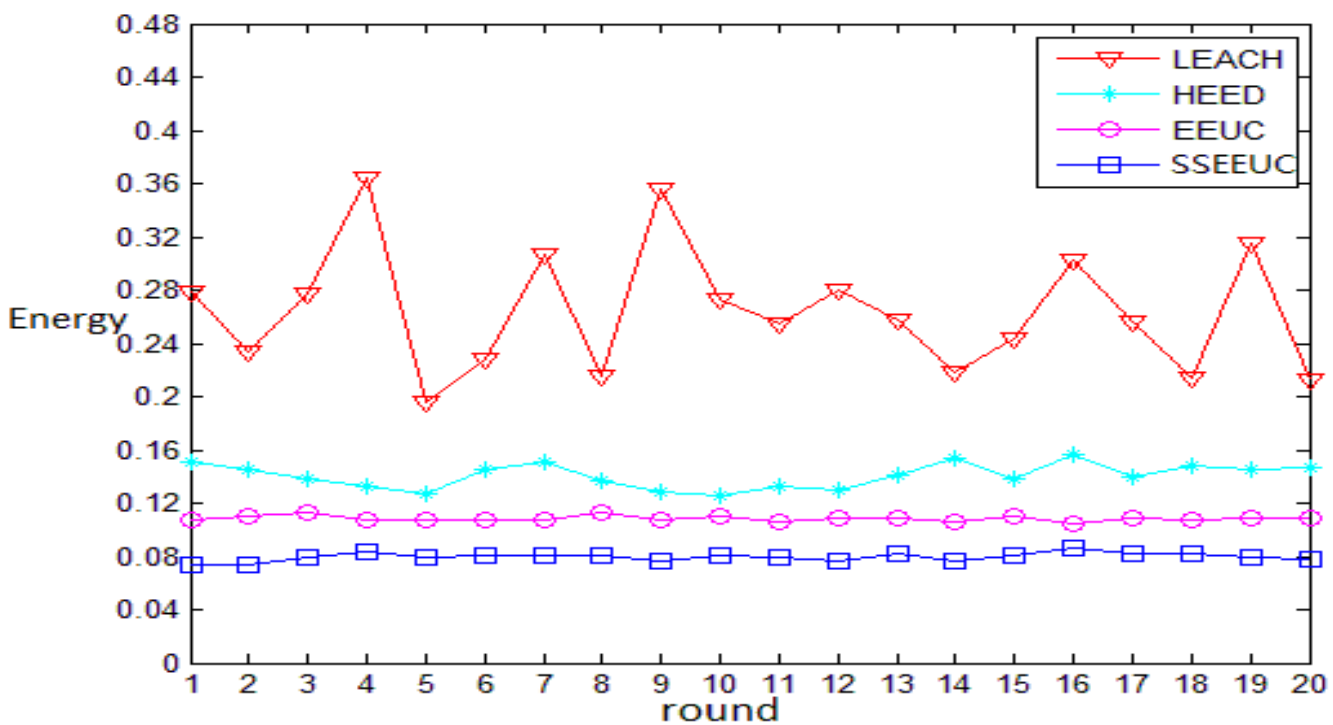

Fig.5 The Sum of the Cluster Heads Energy Consumption

Due to adopting single-hop communication mode between clusters, LEACH consumes more energy than the other three. And the unstable distribution of the clusters number leads to the fluctuation of the energy consumption between round and round. In SSEEUC, we apply the nodes sleep scheduling policy, which makes the number of working ordinary nodes in network is less than the others in general. As a result, the cluster heads can save some energy from the inner-cluster communication cost.

\subsection{The Life Cycle of Network}

The length of the network life cycle is the most intuitive reflect to measure a clustering protocol. In the experiment shown in Figure 6, we simulated the dynamic process of 400 sensor nodes working in a $200 \times 200(\mathrm{~m})$ monitoring area. From Fig6, we can see that the network life cycle of SSEEUC is longer than other three protocols. The reason is that when the data change rate is stable, the neighbor nodes will keep a sleep state reducing energy consumption of the network.

\section{Conclusion and Future Work}

In this paper, we propose a clustering sleep scheduling mechanism SSEEUC based on sentinel nodes monitor for WSN. This protocol is mainly suitable for these application scenarios such as real-time data-aware, target tracking, and environmental areas data collection, etc. We combine the network clustering strategy with the nodes sleep 
scheduling mechanism, and improve the method of selecting the candidate cluster heads randomly in EEUC. Simulation results show that SSEEUC efficiently balances the energy consumption of nodes in wireless sensor networks, and prolongs the network lifetime.

Careful people will find a little detail in Figuer6, the first dead node of SSEEUC is a little earlier than EEUC, we think it's mainly attributes to the randomly distribution way of sensor nodes, As a future work, we plan to prolong the time of first dead node on the basis of SSEEUC.

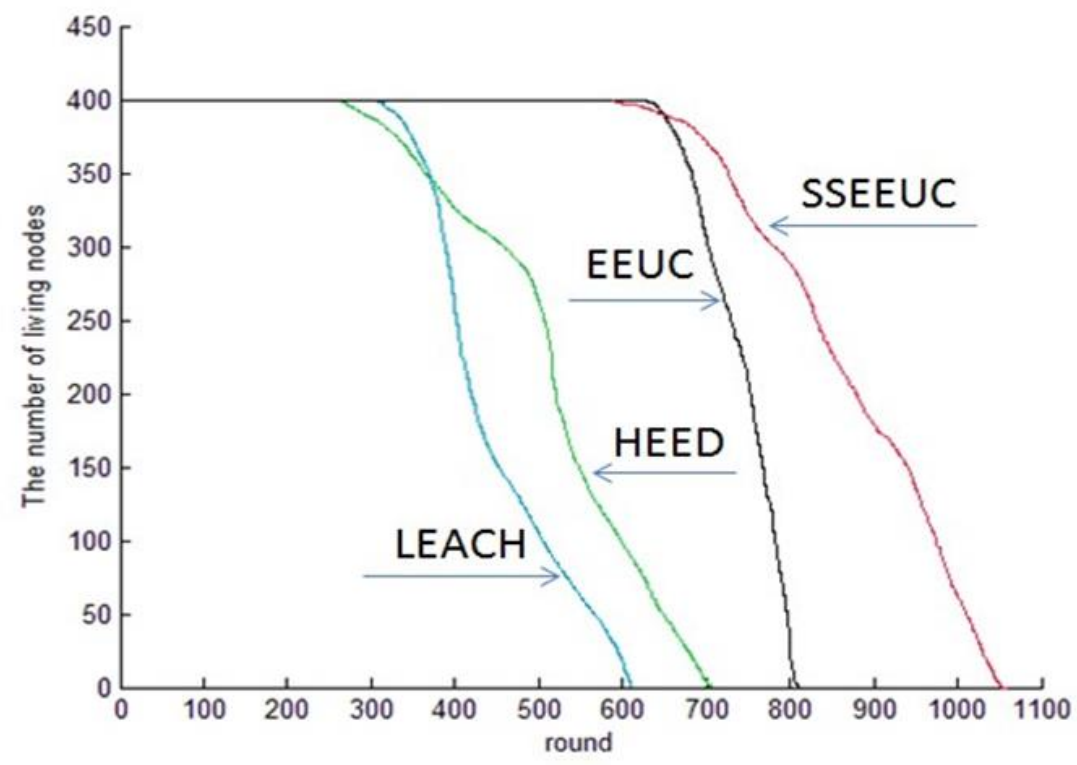

\section{Fig.6 The Number of Alive Sensor Nodes Over Time}

\section{References}

[1] I. F. Akyildiz, W. Su and Y. Sankarasubramaniam, "Wireless sensor networks: a survey", Computer networks, vol. 38, no. 4, (2002), pp. 393-422.

[2] L. M. Sun, "Wireless sensor network (WSN)", Tsinghua University Press, (2005).

[3] Z. S. He, Y. G. Zhu and Y. B. Zhang, "A Survey of Coverage control technology in wireless sensor network", Journal of Computer Measurement \& Control, vol. 20, no. 7, (2012), pp. 1737-1739.

[4] S. B. Xia and E. Xu, "Wireless sensor network explore", Journal of communications technology, (2010), pp. $18-20$.

[5] D. Estrin, "Wireless sensor networks tutorial part IV: sensor network protocols", Mobicom, Westin Peachtree Plaza, Atlanta, Georgia, USA, (2002), pp. 23-28.

[6] J. Hill, R. Szewczyk and A. Woo, "System architecture directions for networked sensors", ACM SIGOPS operating systems review, ACM, vol. 34, no. 5, (2000), pp. 93-104.

[7] W. R. Heinzelman, A. Chandrakasan and H. Balakrishnan, "Energy-efficient communication protocol for wireless micro-sensor networks", System Sciences, Proceedings of the 33rd Annual Hawaii International Conference on IEEE, vol. 2, (2000), p. 10.

[8] W. R. Heinzelman, A. P. Chandrakasan and H. Balakrishnan, "An application-specific protocol architecture for wireless micro-sensor networks", Wireless Communications, IEEE Transactions on, vol. 1, no. 4, (2002), pp. 660-670.

[9] S. Lindsey and C. S. Raghavendra, "PEGASIS: Power-efficient gathering in sensor information systems", Aerospace conference proceedings, IEEE, vol. 3, no. 3, (2002), pp. 1125-1130.

[10] O. Younis and S. Fahmy, "HEED: a hybrid, energy-efficient, distributed clustering approach for ad hoc sensor networks", Mobile Computing, IEEE Transactions on, (2004), vol. 3, no. 4, pp. 366-379.

[11] C. F. Li, M. Ye and G. Chen, "An energy-efficient unequal clustering mechanism for wireless sensor networks", Mobile Ad-hoc and Sensor Systems Conference, IEEE International Conference on. IEEE, vol. 8, (2005), p. 604.

[12] E. Y. Lin, "A comprehensive study of power-efficient rendezvous schemes for wireless sensor networks", University of California, (2005). 
[13] Y. Sun, S. Du and O. Gurewitz, "DW-MAC: a low latency, energy efficient demand-wakeup MAC protocol for wireless sensor networks", Proceedings of the 9th ACM international symposium on Mobile ad hoc networking and computing, ACM, (2008), pp. 53-62.

[14] Q. Cao, T. Abdelzaher and T. He, "Towards optimal sleep scheduling in sensor networks for rare-event detection", Proceedings of the 4th international symposium on Information processing in sensor networks, IEEE Press, (2005), p. 4.

[15] W. Ye, J. Heidemann and D. Estrin, "An energy-efficient MAC protocol for wireless sensor networks", INFOCOM, Twenty-First Annual Joint Conferences of the IEEE Computer and Communications Societies. Proceedings, IEEE, vol. 3, (2002), pp. 1567-1576.

[16] J. Deng, Y. S. Han and W. B. Heinzelman, "Scheduling sleeping nodes in high density cluster-based sensor networks", Mobile Networks and Applications, vol. 10, no. 6, (2005), pp. 825-835.

[17] Y. L. Deng, "The Research of Node Sleep-scheduling Algorithm in Wireless", Chongqing University of posts and telecommunications, (2006).

[18] J. S. Liu, H. P. Lin, "C. Power-efficiency clustering method with power-limit constraint for sensor networks", Performance, Computing, and Communications Conference, Conference Proceedings of the 2003 IEEE International, IEEE, (2003), pp. 129-136 